\title{
A Multi-Agent Scheme for Energy-Efficient Coverage Control in Wireless Sensor Networks
}

\author{
Akkachai Phuphanin and Wipawee Usaha ${ }^{+}$ \\ School of Telecommunication Engineering, Suranaree University of Technology, 111 University Avenue, \\ Muang District, Nakhon Ratchasima 30000 Thailand
}

\begin{abstract}
The underlying aim of this paper is to maximize the energy efficiency in the coverage control scheme in a wireless sensor network (WSN) by selecting the minimal number of working nodes while still maintaining network coverage area. The proposed algorithm is based on a self-adaptive multi-agent system (MAS) coverage control scheme whereby sensor nodes learn to adjust their own coverage to achieve the network-wide coverage. This paper proposes a variation of an existing MAS scheme called the distributed value function (DVF) which differs from the original scheme in the use of cost function which is a function of redundant coverage area. Performance evaluation were compared with a guaranteed complete coverage method, i.e., the optimal geographical density control (OGDC) scheme, and a partial area coverage scheme, i.e., the Probing Environment and Adaptive Sleeping (PEAS) scheme. Results show that modified DVF can achieve a nearly full coverage with only $13-64 \%$ of active sensor nodes whereas the OGDC and PEAS required $14-68 \%$ and $16-76 \%$ of active sensor nodes, respectively for high to low node densities. Results suggests that the MAS coverage control scheme can achieve efficient coverage control, is self-adaptive and therefore suitable for coverage control applications in WSNs such as lighting control in smart offices.
\end{abstract}

Keywords: coverage control, multi-agent systems, wireless sensor networks, reinforcement learning.

\section{Introduction}

Wireless sensor networks (WSNs) are a collection of numerous cheap sensory devices installed within a particular environment to gather the physical parameters of interest. Measurements of these sensor devices are then acquired and relayed through the network to be processed or collected at the base station. Such data acquisition gives the ability to continuously monitor the particular surroundings of interest and respond quickly to any changes that may incur. WSNs have emerged in biomedical, military, agricultural monitoring and control applications [1], [2], [3]. In smart homes or buildings, lighting control have been a particular application which coverage control is needed to reduce energy consumption while maintaining a required level of light intensity.

Coverage control problems have been a significant issues arising in wireless sensor networks with the aim to extend the longevity of network lifetime and efficient energy consumption in the network due to the limited on-board battery power of a sensor node [4]. Refs. [5] and [6] proposed a distributed wireless sensor network with adjustable sensing radii enabling a flexible and efficient coverage. In [7], the authors proposed a Probing Environment and Adaptive Sleeping (PEAS) scheme which is a coverage maintenance scheme that increases the network lifetime by maintaining a necessary number of working nodes and shutting down the rest as reserve. By querying neighboring nodes, a particular working node can determine the status of neighboring working and sleeping nodes prior to deciding on its own status. In [8], the optimal geographical density control (OGDC) scheme was proposed as a guaranteed full coverage control scheme based on grid redundancy check and sequential node activation. The grid redundancy requires that each sensor node

\footnotetext{
+ Corresponding author. Tel.: + (66) 44224392 ; fax: +(66) 44224603.

E-mail address: wusaha@ieee.org.
} 
maintain a list of the grid points it covers. The sequential node activation requires that each active node sends out activation messages to neighboring nodes to reset their timers.

The aforementioned coverage control methods are non-learning schemes which require reconfiguration should the environment change. Due to node deployment in potentially wide areas, direct access to reconfigure the nodes may not be feasible. On the other hand, multi-agent system (MAS) technologies have shown to be promising due to their flexibility and self- adaptability which caters autonomous self-awareness at sensor nodes [11]. In a multi-agent system (MAS), nodes act as agents which have the ability to learn and adjust their coverage in a distributed manner thereby enabling a light weight self-adaptive coverage control. The nodes in a MAS decide their actions in a cooperative manner to achieve a mutual goal of maximizing the network coverage by using the minimal amount of energy. To do so, a cost function based on a function of redundant coverage areas of a sensor node is introduced.

The contribution of this paper is thus twofold: 1) a modified multi-agent coverage control scheme based on a redundancy coverage area cost function; 2) comparison of the scheme with non-learning coverage control schemes, i.e., OGDC and PEAS. The objective is to maximize the coverage control efficiency by maximizing the obtained coverage control per unit of energy consumed. Our results suggests the suitability of applying MAS in coverage control in WSNs.

\section{Multi-agent Coverage Control}

\subsection{Distributed Value Function Scheme}

A multi-agent coverage control scheme called the Distributed Value Function (DVF) has been proposed to co-ordinately and cooperatively improve the coverage control performance in wireless sensor networks [9]. In this method, each node communicates and exchanges information about its value function. A value function is a function that quantifies how well the agent (sensor node) performs at a given state $s \in S$ where $S$ is a discrete set of all possible states of the sensor network. Let $a \in A$ be the action selected by an agent, where $A$ is the discrete set of all possible actions available at each state. The decision rule of an agent, so called policy $\pi$, is defined as a rule which the agent selects an action as a function of its state. In other words, it is the mapping from a state $s \in S$ and action $a \in A$ to the probability of selecting action $a$ at state $s$. The value function of state $s$ under a given policy $\pi$ is formally defined by $V^{\pi}(s)=E^{\pi}\left\{\sum_{k=0}^{\infty} \gamma^{k} r_{t+k+1} \mid s_{t}=s\right\}$, where $r_{t+1}$ is the reward of taking a particular action in a given state $s$ at time $t, \gamma$ is the discount factor and $E^{\pi}\{\}$ is the expectation operator. Similarly, we define the action value function of taking action $a$ at a given state under policy $\pi$ by

$$
Q^{\pi}(s, a)=E^{\pi}\left\{\sum_{k=0}^{\infty} \gamma^{k} r_{t+k+1} \mid s_{t}=s, a_{t}=a\right\}
$$

The objective is to find a policy $\pi^{*}$ such that $\pi^{*}=\underset{\forall \pi}{\operatorname{argmax}} Q^{\pi}(s, a)$. To achieve this objective, each agent $i$ (node) in the DVF algorithm performs an update of its own action value function. The update rule at time step $t$ for agent $i$ is given by [9]:

$$
\begin{gathered}
Q_{t+1}^{i}\left(s_{t}^{i}, a_{t}^{i}\right)=(1-\alpha) Q_{t}^{i}\left(s_{t}^{i}, a_{t}^{i}\right)+\alpha\left(r_{t+1}^{i}\left(s_{t+1}^{i}\right)+\gamma \sum_{j \in N e i g h(i)} f^{i}(j) V_{t}^{j}\left(s_{t+1}^{j}\right)\right) \\
V_{t+1}^{i}\left(s_{t}^{i}\right)=\max _{a \in A^{i}} Q_{t+1}^{i}\left(s_{t}^{i}, a\right)
\end{gathered}
$$

where $\alpha$ is the learning rate, $f^{i}(j)$ are factors that weigh the value functions of the neighbors of agent $i$ such that

$$
f^{i}(j)=\left\{\begin{array}{cc}
\frac{1}{|\operatorname{Neigh(}(i)|} & , \text { if } \operatorname{Neigh}(i) \neq 0 \\
1 & \text {, otherwise }
\end{array}\right.
$$

where $j \in \operatorname{Neigh}(i)$ is the set of neighbors of node $i[9]$. 


\subsection{Modified DVF Framework for Coverage Control}

Consider a wireless sensor network comprising multiple light sensor nodes. For a particular sensor node $i$, the local state and actions taken are defined as follows.

Local agent state: Each sensor node $i$ can sense the level of coverage in its area. Its local state $s^{i}$ is state of each agent based on its mode and coverage area.

Local agent actions: Each sensor node $i$ has the ability to take one of the following actions in any state it lands in. The action space $A^{i}$ is the set of all possible actions for each state $A^{i}=\{$ Action_0, Action_1 $\}$ where Action_O (Action_l) refers to sensor node $i$ turning off (on).

Each action decided by sensor node $i$ results in a reward, denoted as $r^{i}\left(s_{t}^{i}\right)$ which is a function of sensor node $i$ 's state $s^{i}$ at time $t$ defined by

$$
r^{i}\left(s_{t}^{i}\right)=G^{i}\left(s_{t}^{i}\right)-C^{i}
$$

where $G^{i}\left(s_{t}^{i}\right)$ is a function of the number of cells within the coverage area of sensor node $i$ such that

$$
G^{i}\left(s_{t}^{i}\right)=\text { Area_coverage }\left(a^{i}\right) \times G A I N_{-} C E L L_{-} B R I G H T,
$$

and $C^{i}$ is the area overlapped as a result from the action taken by sensor node $i$ at time $t$.

\section{Performance Evaluation}

To compare the coverage control performance of a WSN, we considered a gridded area of $1000 \times 1000$ sq.m. containing a number of sensor nodes ranging from 100,200,300,400,500 sensor nodes placed randomly in the area.

The objective is for the sensor node to learn to cooperate with one another in order to completely coverage area in an energy-efficient way, i.e. minimize the number of sensor node turned on. The coverage area of node (agent) $i$ was given within a transmission range of $100 \mathrm{~m}$. The initial energy of each sensor node was 10 Joule. Comparison was based on the number of working nodes, coverage percentage and coverage lifetime. The DVF was compared with OGDC, PEAS8 and PEAS9, where the latter two are PEAS with probing ranges of 80 and $90 \mathrm{~m}$, respectively.

According to equations (1) and (4), the value of the learning rate $\alpha=0.4$, the discount factor $\gamma=0.7$ and the GAIN_CELL_BRIGHT $=0.5$. The values of the learning rate and discount factor were obtained from experimenting a range of values and selecting the parameters which received the best performance in terms of average accumulated reward in (4). The simulation results were averaged over 10 runs to achieve the desired accuracy.

Fig 1 depicts the number of working nodes against the number of deployed nodes in each algorithm. Note that OGDC, PEAS8 and PEAS9 consistently use a gradually increasing number of working nodes which is higher than DVF. The reason is due to DVF determines the working nodes which achieves the best coverage while saving energy consumption.

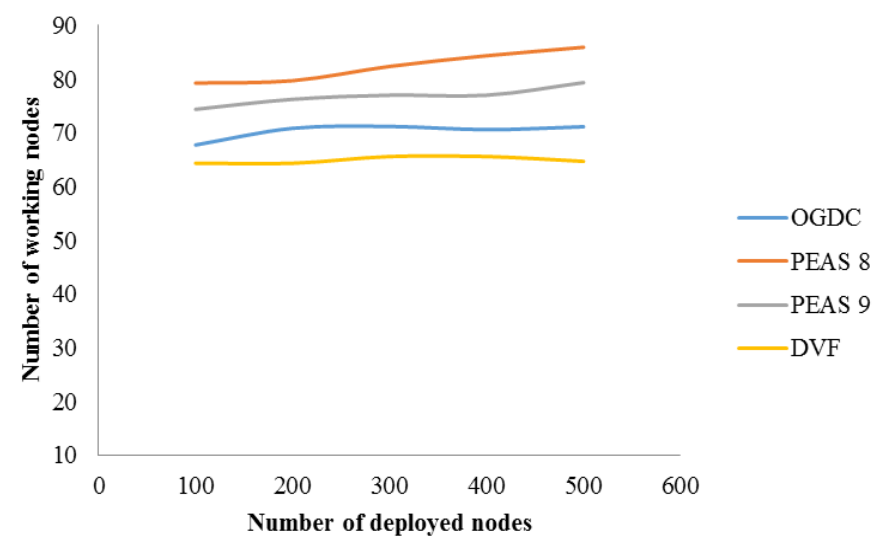

Fig. 1: Average number of working nodes against the number of deployed nodes. 
Fig 2 shows the percentage of coverage achieved by all algorithms against the number of deployed nodes. Note that above 200 deployed nodes, all algorithms can achieve full coverage with DVF attaining 99.2\% coverage at 200 nodes and $99.8 \%$ at 500 deployed nodes. The reason is because DVF must conservatively select working nodes so as to reduce the amount of energy consumption. Even so, the modified DVF can achieve a nearly full coverage with only $13-64 \%$ of active sensor nodes whereas the OGDC and PEAS required $14-68 \%$ and $16-76 \%$ of active sensor nodes, respectively for high to low node densities.

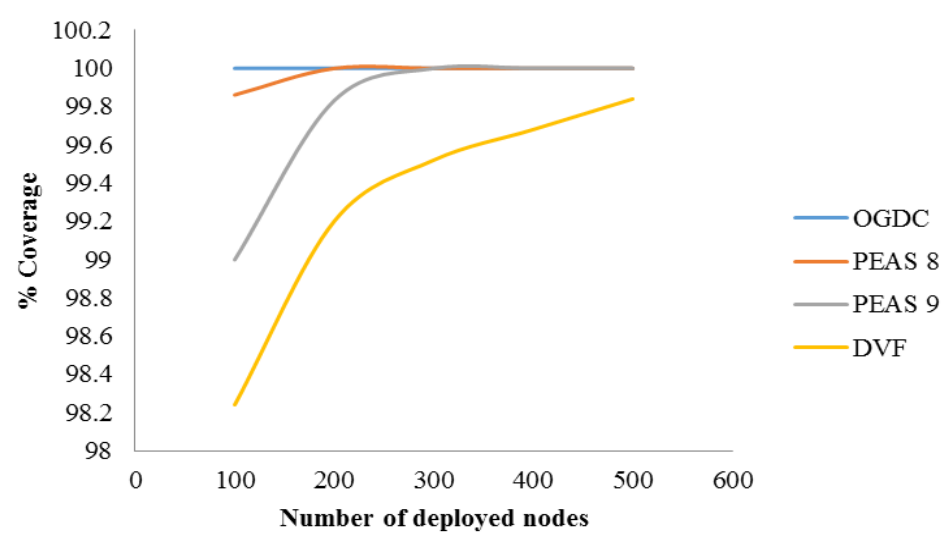

Fig. 2: Percentage of coverage against the number of deployed nodes.

Fig. 3. illustrates the efficiency in terms of coverage area per working node for each algorithm. The rational is from the fact that coverage area attained is a trade-off with the energy dissipated by the working node. Results show that DVF achieved the highest coverage per working node, followed by OGDC and PEAS.

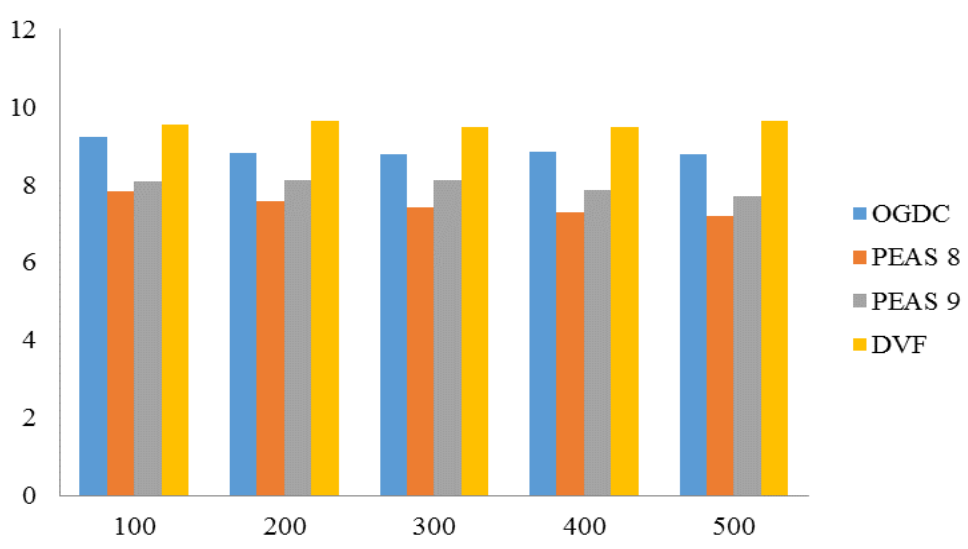

Fig. 3: Average coverage area per working node against number of deployed nodes.

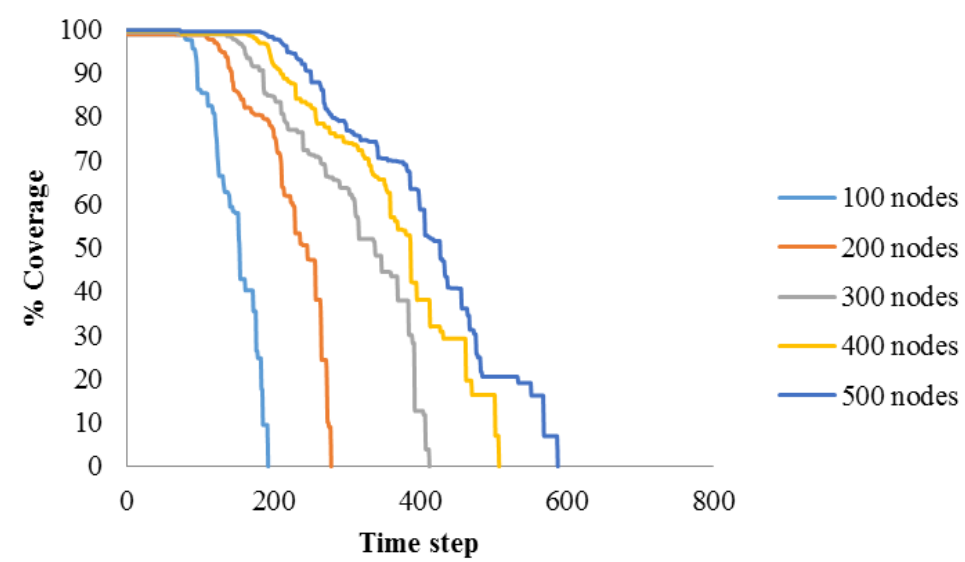

Fig. 4: Coverage lifetime for DVF. 
Fig. 4,5,6 depict the area coverage lifetime for each algorithm for various numbers of deployed nodes. The area coverage lifetime is defined by the duration from the start of network operation until the coverage requirement is no longer satisfied. It is evident that the coverage lifetime for DVF is prolonged the most in terms of number of time steps due to the least number of working nodes. PEAS8 attained the least coverage lifetime. This is because PEAS requires acknowledgement messages in addition to the higher number of working nodes than OGDC and DVF.

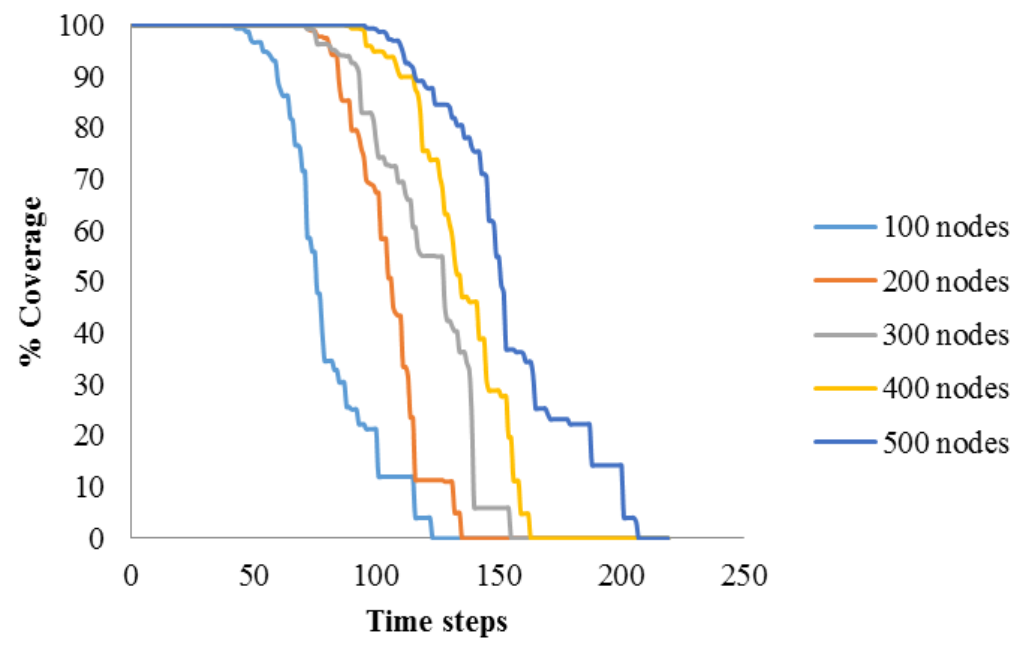

Fig. 5: Coverage lifetime for OGDC.

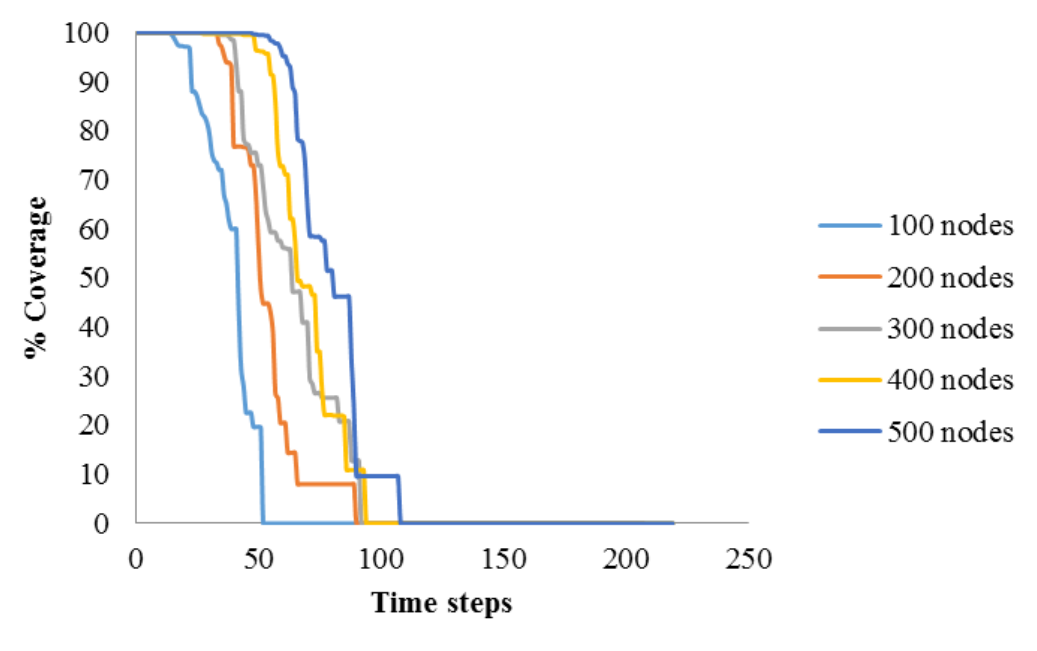

Fig. 6: Coverage lifetime for PEAS8.

\section{Conclusions}

In this paper, we have proposed a cost function-modified distributed value function (DVF) scheme which is a multi-agent scheme aimed at energy-efficient coverage control in wireless sensor networks. Results were compared with two non-learning coverage control schemes i.e., PEAS which is a partial coverage control scheme and OGDC which is a guaranteed coverage control scheme. Results showed that the proposed modified DVF attained the least working nodes of all while still achieving nearly complete coverage. Therefore, DVF outperformed PEAS and OGDC in terms of area coverage energy efficiency and area coverage lifetime. Results suggest the suitability of employing MAS for coverage control problems in WSNs. In the future, we plan to apply the DVF framework to lighting control applications in smart offices.

\section{Acknowledgements}

This research is supported by the National Research Council of Thailand. 


\section{References}

[1] A. Alaiad and L. Zhou, "Patients' Behavioral Intentions toward Using WSN based Smart Home Healthcare Systems: An Empirical Investigation," Proceedings of the 2015 Hawaii International Conference on System Sciences (HICSS), p.824-833, January, 2015.

[2] Md.A.Hussain, P. Khan, and K.K. Sup," WSN Research Activities for Military Application”, Proceedings of 2009 International Conference on Advanced Communication Technology, p.271-274, February, 2009.

[3] Santoshkumar, and Udaykumar R.Y, "Development of WSN System for Precision Agriculture", Innovations in Information, Embedded and Communication Systems (ICIIECS), 2015 International Conference on, p.1-5, March 2015.

[4] B. Wang, "Coverage Problems in Sensor Networks: A Survey," ACM Computing Surveys, 43(4), Article 32, 53 pages, October, 2011.

[5] C. T. Vu, Z. Cai, and Y. Li, "Distributed Energy-Efficient Algorithms to Maximize Network lifetime for Coverage Problem in Adjustable Sensing Radii Wireless Sensor Networks", submitted to Discrete Mathematics, Algorithms and Applications (DMAA).

[6] C. T. Vu, G. Chen, Y. Zhao, and Y. Li, "A Universal Framework for $\alpha$-Coverage Problem in Wireless Sensor Network", Proceedings of the 2009 IEEE International Conference on Network Protocols (ICNP).

[7] F. Ye, H. Zhang, S. Lu, L. Zhang and J. Hou, "A Randomized Energy-Conservation Protocol for Resilient Sensor Networks,” Wireless Networks ,12(5), p.637-652, 2006.

[8] H. Zhang, J. C. Hou, "Maintaining Sensing Coverage and Connectivity in Large Sensor Networks", Ad Hoc \& Sensor Wireless Networks, 1(1), pp.89-124, 2005.

[9] C.K. Tham, and J.C. Renaud, "Multi-Agent Systems in Sensor Networks: A Distributed Reinforcement Learning Approach", Proceedings of the 2005 International Conference on Intelligent Sensors, Sensor Networks and Information Processing, December, 2005.

[10] A. Phuphanin and W. Usaha, "Secure Coverage Control in Wireless Sensor Networks with Malicious Nodes using Multi-Agents" Proceedings of the 2011 IFIP International Conference on Embedded and Ubiquitous Computing (EUC), October, 2011.

[11] M Vinyals, JA Rodriguez-Aguilar, J. Cerquides, "A Survey on Sensor Networks from a Multiagent Perspective," The Computer Journal, 54(3), p.455-470, 2011. 